\title{
DESIGN AND MODELLING OF A COMPLIANT CONSTANT-FORCE SURGICAL TOOL FOR OBJECTIVE ASSESSMENT OF OSSICULAR CHAIN MOBILITY \\ Loïc Tissot-Daguette ${ }^{1 *}$, Charles Baur ${ }^{1}$, Axel Bertholds ${ }^{2}$, Pere Llosas ${ }^{2}$, and Simon Henein ${ }^{1}$ \\ ${ }^{1}$ Instant-Lab, EPFL, Lausanne, SWITZERLAND and \\ ${ }^{2}$ Sensoptic SA, Losone, SWITZERLAND
}

\begin{abstract}
Surgeons generally assess the mobility of the ossicle chain by manual palpation using rigid elongated hooks inserted through the ear canal. As the applied forces are in the range of a few grams equivalent force, the measurement remains subjective and its reliability highly dependent on the level of experience and skills of the surgeon. This paper presents the design, modelling and experimental testing of an innovative surgical device based on a novel compliant constant-force mechanism used to apply a predefined force of $1 \mathrm{gf}(9.81 \mathrm{mN})$ to the ossicles. Using this handheld constant-force tool along with an operating microscope for visual feedback of ossicle displacement, surgeons can objectively quantify ossicular mobility.
\end{abstract}

\section{KEYWORDS}

Constant-force mechanism, Compliant mechanism, Beam buckling, Handheld force limiting device, Surgical instrument, Ossicular chain mobility, Otology.

\section{INTRODUCTION}

Middle ear sound transmission can be estimated by evaluating the stiffness of each ossicle [1]. Otologists usually assess the ossicular chain mobility by manual palpation with a stiff tool. However, the palpation forces are in the order of 3-15 gf ( 1 gf corresponding to $9.81 \mathrm{mN}$ ) [2]. Thereby, the judgment of normal or impaired mobility of middle ear ossicles is highly dependent on the surgeon's subjective experience.

Current sensors exist to quantitatively measure the ossicular mobility. For example, the optical force-sensing instrument PalpEar can measure the force applied to the ossicles and based on the ossicle displacement, visualized under an operating microscope, ossicular mobility can then be estimated [3]. The device Otopen uses a linear actuator coupled to a highly sensitive load cell to measure ossicle compliance [4]. While, Koike et al. propose a vibrating probe that administers an oscillating displacement on the ossicles and measures the contact force with a load cell [5].

In this paper, we investigate a novel surgical instrument designed for ossicle mobility assessment, which compared to existing active solutions, is entirely passive (i.e., no sensors or actuators are required). The tool contains a compliant constant-force mechanism at its tip that applies a defined force to the ossicle. Analytical modelling of the mechanism based on Euler-Bernoulli beam theory is presented and verified by finite element analyses. We fabricated prototypes of the mechanism from glass using $3 \mathrm{D}$ femtolaser printing and tested them to demonstrate the effectiveness of the constant-force output.

\section{MECHANISM DESCRIPTION}

The proposed handheld tool is presented in Fig. 1. The instrument tip is a compliant constant-force mechanism made of a straight but bendable beam and a flexure pivot (Fig. 2). The way to assess the ossicular mobility using this constant-force tool is as follows: the surgeon progressively increases the force applied to the palpated ossicle until the tool tip's beam reaches its critical load and buckles, thus limiting the force to the respective predefined threshold. The resulting ossicle displacement is then evaluated visually under an operating microscope (for example with the help of another surgical tool that has a ruler placed at its tip). The amplitude of the visualized displacement informs the surgeon on the mobility of the ossicle. Indeed, when the tool applies a constant force $F_{\text {tool }}$, the ossicle displacement $y_{\text {ossicle }}$ depends directly on the ossicle stiffness $K_{\text {ossicle }}$ (represented schematically by a linear spring in Fig. 2), regardless of the handle position $y_{\text {tool }}$.

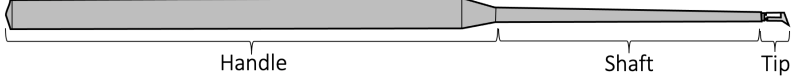

Figure 1: Proposed mechanical device to apply a constant force on ossicles.

Compared to other constant-force mechanisms in the literature [6], our design of simple elastic structures, can be made very compact, requires no preloading and needs only minimum assembly. The instrument is also wear-free, scalable, and sterilizable.

(a)

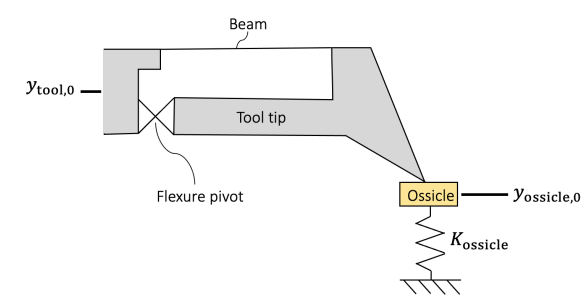

(b)

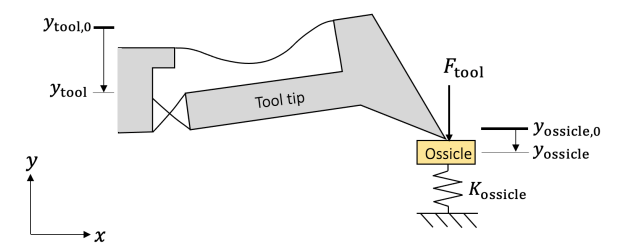

Figure 2: Schematic close-up view of the tool tip palpating an ossicle. (a) The tool tip touches the ossicle without applying force. (b) When the beam buckles, the tool tip is applying a near-constant force to the ossicle.

\section{ANALYTICAL MODEL}

In this section, the analytical modelling is conducted to design the mechanism of the tool tip. Figure 3 shows the deformation and the load case of the compliant mechanism. The initially straight beam is clamped at one end and attached to a rigid lever pivoting around a flexure pivot. The flexure pivot is symbolized by a hinged joint in parallel to a torsional spring having a rotation stiffness $K_{\alpha}$. When 
the lever rotates by an angle $\alpha$, the elastic reactions from the beam and the spring pivot apply a quasi-constant torque $M_{\alpha}$ on the lever. Using the moment-angle relation and the geometry of the lever, a force $F_{\text {tool }}$ can be applied to a vertical linear stage (representing the ossicle) with displacement $\Delta y$.

(a)

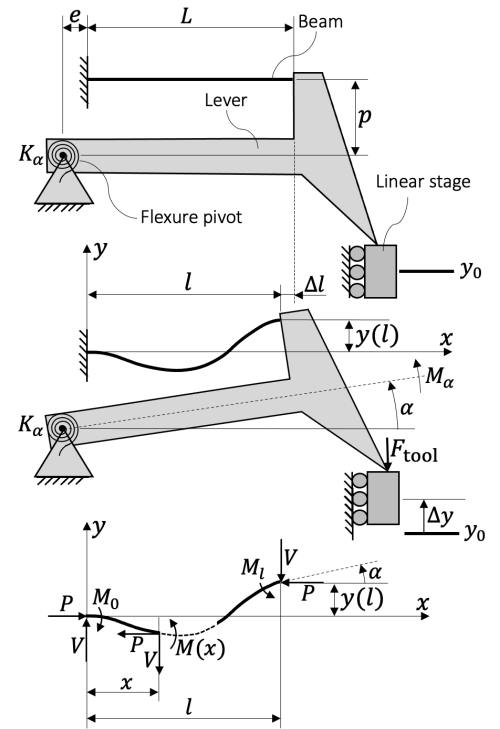

Figure 3: (a) As-fabricated tool tip, (b) deformed tool tip and (c) free-body diagram of the beam.

The buckled beam and the blades of the flexure pivot are considered as initially straight slender beams made of an isotropic linear elastic material. Assuming small beam deformations, the beam deflection can be obtained from Euler-Bernoulli equation:

$$
M(x)=E I y^{\prime \prime}(x)
$$

where $E I$ is the beam flexural rigidity and $M(x)$ is the bending moment related to the beam's reactions as follows:

$$
M(x)=-P y(x)+V x+M_{0}
$$

Combining Eqs. (1) and (2) gives a second-order differential equation. After deriving the general solution and integrating the boundary conditions $y(0)=y^{\prime}(0)=0$, we obtain the following beam deflection:

$$
y(x)=A(\sin (k x)-k x)+B(\cos (k x)-1)
$$

where $k=\sqrt{P / E I}$. The parameters $A$ and $B$ are found using the boundary conditions $y(l)=(L+e) \sin (\alpha)-$ $p(1-\cos (\alpha)) \cong(l+e) \alpha$ and $y^{\prime}(l) \cong \alpha$ :

$$
\begin{gathered}
A=-\alpha l \frac{k l \sin (k l)+\cos (k l)-1+\bar{e} k l \sin (k l)}{k l(k l \sin (k l)+2(\cos (k l)-1))} \\
B=-\alpha l \frac{k l \cos (k l)-\sin (k l)+\bar{e} k l(\cos (k l)-1)}{k l(k l \sin (k l)+2(\cos (k l)-1))}
\end{gathered}
$$

where $\bar{e}=e / l$.
The horizontal displacement $\Delta l$ corresponds to the imposed end-shortening of the beam, which can be calculated from [7]. Neglecting the horizontal contraction of the beam due to the load $P$, we get:

$$
\Delta l=L-l \cong \int_{0}^{l} \frac{\left(y^{\prime}(x)\right)^{2}}{2} d x=H(k l) l \alpha^{2}
$$

where:

$$
\begin{aligned}
H(k l)= & \frac{k l}{8}\left[\left(\frac{A}{l \alpha}\right)^{2}(6 k l-8 \sin (k l)+\sin (2 k l))\right. \\
& +2\left(\frac{A}{l \alpha}\right)\left(\frac{B}{l \alpha}\right)(\cos (2 k l)-4 \cos (k l)+3) \\
& \left.+\left(\frac{B}{l \alpha}\right)^{2}(2 k l-\sin (2 k l))\right]
\end{aligned}
$$

This horizontal displacement is linked to the rotation of the lever with the approximated relation:

$$
\begin{gathered}
\Delta l=(L+e)(1-\cos (\alpha))+p \sin (\alpha) \\
\cong(l+e) \frac{\alpha^{2}}{2}+p \alpha
\end{gathered}
$$

After equalizing Eqs. (6) and (8), we derive the non-trivial solution of the angle $\alpha$ :

$$
\alpha \cong \frac{\bar{p}}{H(k l)-\frac{(1+\bar{e})}{2}}
$$

where $\bar{p}=p / l$.

The moment applied on the lever is given by:

$$
\begin{aligned}
M_{\alpha}= & M_{l}+P(p+y(l))-V(l+e)+K_{\alpha} \alpha \\
= & \frac{E I}{l} \bar{p}\left((k l)^{2}+J(k l)\right)+K_{\alpha} \alpha
\end{aligned}
$$

where:

$$
\begin{aligned}
& J(k l)= \\
& k l \frac{k l \cos (k l)-\sin (k l)\left(1+\bar{e}(k l)^{2}+\bar{e}^{2}(k l)^{2}\right)}{\left(H(k l)-\frac{(1+\bar{e})}{2}\right)(k l \sin (k l)+2(\cos (k l)-1))}
\end{aligned}
$$

Finally, the force applied at the tool tip can be expressed as:

$$
F_{\text {tool }}=\frac{M_{\alpha}}{D \cos (\alpha)+d \sin (\alpha)}
$$

where $d$ and $D$ are respectively, the vertical and horizontal distances between the flexure pivot axis of rotation and the point where the force is applied when the mechanism is undeformed (see Fig. 4). The vertical displacement of the tool tip is equal to:

$$
\Delta y=D \sin (\alpha)+d(1-\cos (\alpha))
$$

Based on the analytical model, we propose a design of the mechanism to obtain a near-constant force of $1 \mathrm{gf}$. The design parameters are given in Table 1. 


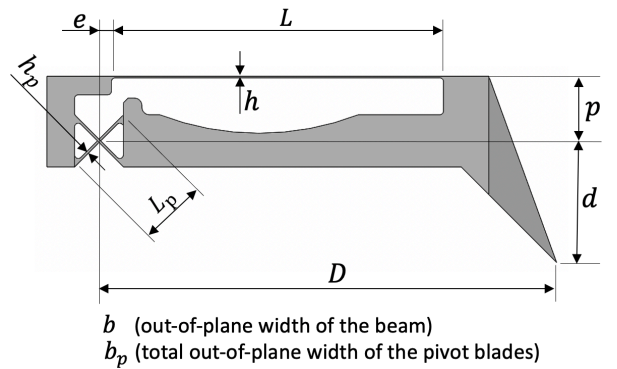

Figure 4: Design of the tool tip (illustrated as-fabricated).

The material of the flexible tool tip was chosen as glass (fused silica). This material was selected for its biocompatibility and its high yield strength on Young's modulus ratio $\left(\sigma_{y} / E\right)$ if previously etched in an HF bath [8]. The flexure pivot was a cross-spring pivot made of three separate blades. Its rotation stiffness is given by [9]:

$$
K_{\alpha}=\frac{E b_{p} h_{p}^{3}}{12 L_{p}}
$$

Table 1: Design parameters of the mechanism (see Fig. 4).

\begin{tabular}{|l|c|c|}
\hline & Parameter & Value \\
\hline Material & $E$ & $74 \mathrm{GPa}$ \\
(fused silica) & $\sigma_{y}$ & $800 \mathrm{MPa}$ \\
\hline Beam & $h$ & $16 \mu \mathrm{m}$ \\
& $b$ & $1 \mathrm{~mm}$ \\
& $L$ & $3.53 \mathrm{~mm}$ \\
\hline Lever & $p$ & $0.7 \mathrm{~mm}$ \\
& $e$ & $0.175 \mathrm{~mm}$ \\
& $D$ & $5 \mathrm{~mm}$ \\
& $d$ & $1.3 \mathrm{~mm}$ \\
\hline Flexure pivot & $h_{p}$ & $25 \mu \mathrm{m}$ \\
& $b_{p}$ & $0.8 \mathrm{~mm}$ \\
& $L_{p}$ & $0.65 \mathrm{~mm}$ \\
& $K_{\alpha}$ & $0.119 \mathrm{Nmm}$ \\
\hline
\end{tabular}

\section{FEM VERIFICATION}

To validate our analytical model and design, we carried out a finite element method (FEM) 3D static study using Comsol Multiphysics 5.4 considering geometric nonlinearities. A first study was conducted to confirm that the material could sustain the internal stress when the structure is deformed to its full stroke. Figure 5 shows that the maximum von Mises stress in the structure is $507 \mathrm{MPa}$ and $394 \mathrm{MPa}$ when the beam buckles upwards and downwards, respectively. These values are below the material yield stress stated in Table 1 .

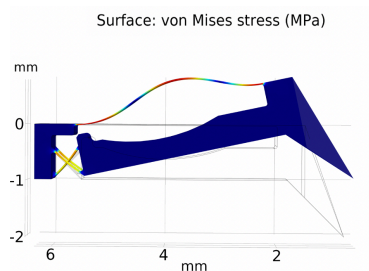

(a)

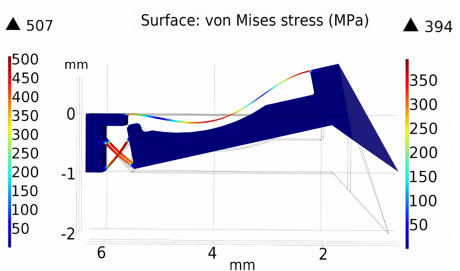

(b)
Figure 5: FEM simulation of the design. A $1.07 \mathrm{~mm}$ displacement is applied to the tool's tip when the beam buckles (a) upwards and (b) downwards.
Figure 6 illustrates the simulated force-displacement characteristics of the tool tip mechanism. From Eqs. (12) and (13), the analytical curve $\left(F_{\text {tool }}\right.$ versus $\left.\Delta y\right)$ is plotted in Fig. 6 as the parameter $k l$ varies around $2 \pi(k l=2 \pi$ corresponding to the Euler's critical load of a clampedclamped beam [10]), with the design parameters stated in Table 1 and the approximation $l \cong L$. When $k l>2 \pi$, the beam buckles upwards (Fig. 5(a)) and $k l<2 \pi$ refers to the downwards buckling of the beam (Fig. 5(b)). Hence, $k l=$ $2 \pi$ is a bifurcation point where the undeformed beam could buckle upwards or downwards. The preferable beam deflection is downwards because, as seen in Fig. 6, the output force is nearly constant.

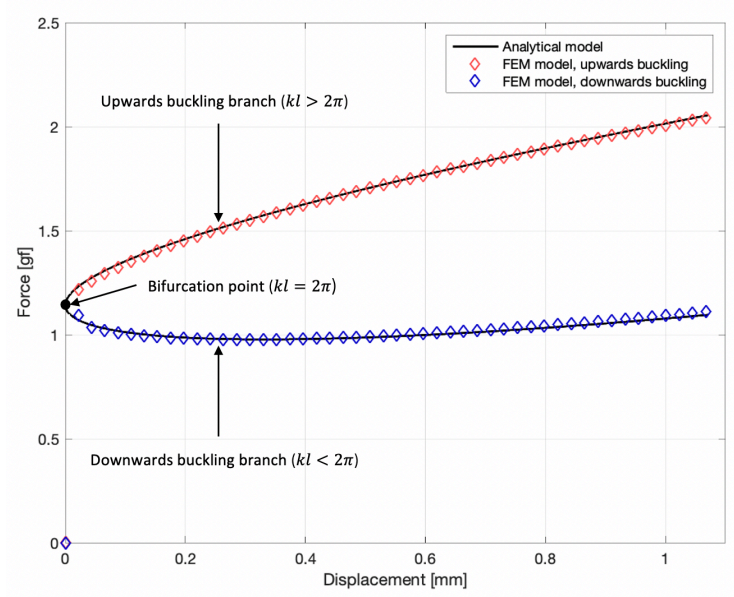

Figure 6: Force-displacement characteristics of the mechanism (analytical model and FEM simulations).

\section{EXPERIMENTAL RESULTS}

In order to prove the validity of the analytical and FEM models, four prototypes with the dimensions stated in Table 1 were fabricated in fused silica using femtolaser 3D printing technology. The tool shaft and handle were made out of biocompatible stainless steel (316L) to obtain a high rigidity compared to the flexible glass tool tip. The tool parts were assembled using biocompatible and sterilizable epoxy. A testbench was used to characterize the force versus displacement relation of the four samples. The components of the experimental setup are depicted in Fig. 7. The tool handle is attached to a manual micrometer linear stage to apply a defined vertical displacement to the tool tip, in contact with a precision balance (Mettler Toledo PR5003 DualRange) used to measure the contact force.

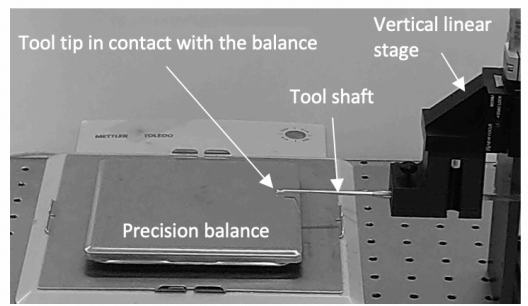

Figure 7: Experimental setup to measure the forcedisplacement characteristics of the constant-force tool.

During testing, the beam of the three first prototypes always buckled upwards (Fig. 8(a)) compared to the fourth sample, which constantly buckled downwards (Fig. 8(b)). 


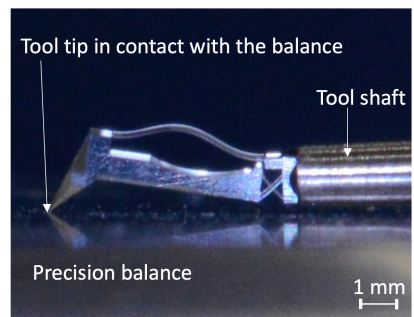

(a)

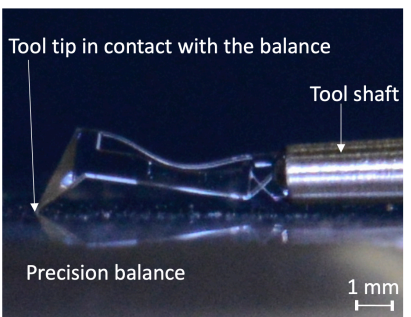

(b)
Figure 8: Tool tip deformation during force-displacement characteristics testing with (a) a prototype buckling upwards and (b) a prototype buckling downwards.

The experimental results are plotted in Fig. 9 with \pm 0.05 gf error bars, along with the analytical curve. Out of the four prototypes, one sample (sample 4) showed promising results with a quasi-constant force of $1 \mathrm{gf}$ with 0.1 gf maximum variation in a stroke range of $0.87 \mathrm{~mm}$. Extremely small tool tip displacement (less than $0.01 \mathrm{~mm}$ ) was observed before reaching the constant-force region.

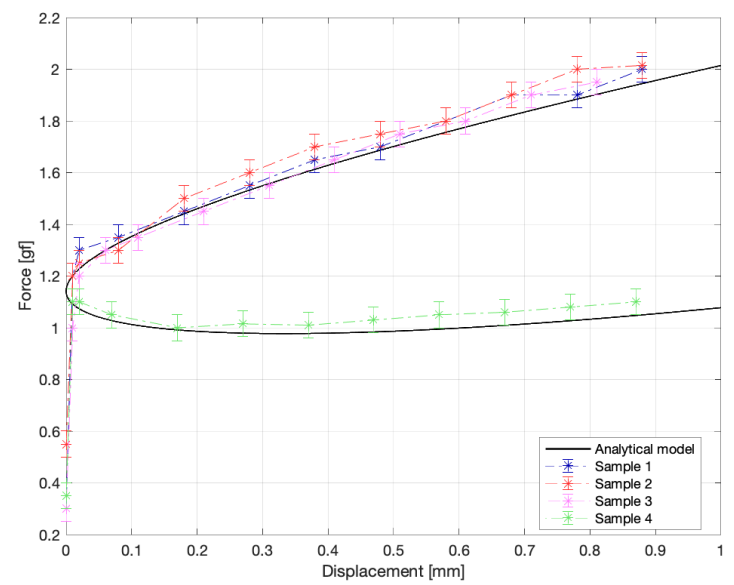

Figure 9: Force-displacement characteristics of the mechanism (analytical model and experimental results).

\section{DISCUSSION}

Very small deviations were observed between the analytical and FEM models, which validates the analytical model proposed in this paper. A minor discrepancy offset between the analytical and experimental data is assumed to be due to manufacturing tolerances.

Even though the manufacturing process seemed rather repeatable, the samples showed different beam buckling directions. We suppose that the buckling direction is linked to the fabrication tolerance of the beam's initial curvature. Based on this assumption, five new prototypes with slightly pre-curved beams were built. Tests revealed that all the new samples had a beam that always buckles downwards, resulting in a constant force output, as desired.

A main drawback of the new tool could be the brittleness of the glass tool tip. Possible protection might be added to protect the tool tip from undesired collisions to avoid any sharp glass parts dropping into the patient's middle ear. Non-brittle materials such as plastic and metal could be investigated for a flexible-structured tool tip.

The buckling-based mechanism may be adaptable to other applications where constant-force is necessary (e.g., robot end-effectors, MEMS, etc.).

\section{CONCLUSION AND FUTURE WORK}

We designed a novel compliant constant-force tool, modelled it and validated it experimentally in this paper. The results revealed that prototypes made out of glass can yield a quasi-constant force of $1 \mathrm{gf}$ with a $0.1 \mathrm{gf}$ maximum variation over a displacement range of $0.87 \mathrm{~mm}$. This handheld constant-force tool, along with an operating microscope for visual feedback of ossicle displacement, allows surgeons to objectively measure the ossicular chain mobility. The next phase of the research will involve in vivo tests by surgeons. Based on the tests, the design of the tool may be adjusted to obtain the optimized force output.

\section{ACKNOWLEDGEMENTS}

This research was funded by Innosuisse (Project No. 46567.1 INNO-LS). The authors acknowledge FEMTOprint for the manufacture of the fused silica prototypes. The authors also thank Prof. T. Linder, Cantonal Hospital Luzern, Switzerland; Prof. A. Huber, University Hospital Zürich, Switzerland; and Prof. M. Neudert and T. Stoppe, both from Dresden University of Technology, Germany, for advice and testing of the tool.

\section{REFERENCES}

[1] I. Dobrev, J. Sim, B. Aqtashi, A. Huber, T. Linder, C. Röösli, "Effects of middle ear quasi-static stiffness on sound transmission quantified by a novel 3-axis optical force sensor", Hearing Research, vol. 357, pp. $1-9,2018$.

[2] T. Linder, G. Volkan, E. Troxler, “Objective Measurements of Ossicular Chain Mobility Using a Palpating Instrument Intraoperatively", Otology \& Neurotology, vol. 36, pp. 1669-1675, 2015.

[3] A. Bertholds, P. Llosas, S. Henein, "Optical force sensing element and microsurgical instrument", Patent Application, US 2013/0204142 A1.

[4] J. Casey, "Diagnostic tool for measuring ossicular chain compliance", Patent Application, WO 2015/168698 A1.

[5] T. Koike, M. Murakoshi, S. Hamanishi, U. Yuasa, R. Uasa, T. Kobayashi, H. Wada, "An apparatus for diagnosis of ossicular chain mobility in humans", Int. Journal of Audiology, vol. 45, pp. 121-128, 2006.

[6] P. Wang, Q. Xu, "Design and modeling of constantforce mechanisms: A survey", Mechanism and Machine Theory, vol. 119, pp. 1-21, 2018.

[7] M. Vangbo, "An analytical analysis of a compressed bistable buckled beam", Sensors and Actuators, A 69, pp. 212-216, 1998.

[8] Y. Bellouard, "On the bending strength of fused silica flexures fabricated by ultrafast lasers", Optical Materials Express, vol. 1, no. 5, pp. 816-831, 2011.

[9] F. Cosandier, S. Henein, M. Richard, L. Rubbert, "The Art of Flexure Mechanism Design", EPFL Press, Lausanne, 2017.

[10] A. Chajes, "Principles of Structural Stability Theory", Civil Engineering and Engineering Mechanics Series, pp. 8-10, 1974.

\section{CONTACT}

*Loïc Tissot-Daguette, loic.tissot-daguette@epfl.ch 